\title{
SOME NEW METHODS OF SOLUTION OF TWO- DIMENSIONAL PROBLEMS IN ELASTICITY
}

\author{
IVAN S. SOKOLNIKOFF
}

1. Introduction. The main purpose of this address is to bring to the attention of the workers in the theory of elasticity and related branches of applied mathematics a simple general method of solution of several important classes of two-dimensional boundary value problems. I use the term "two-dimensional" or "plane" boundary value problems in the sense that their mathematical formulation requires the introduction of only two independent variables. In this sense the problems of St. Venant on torsion and flexure of cylinders, and the problems on deflection and buckling of elastic plates, which have a three-dimensional physical aspect, are two-dimensional.

The method which I intend to discuss was developed mainly by a group of Russian mathematicians, and despite the fact that it has been utilized extensively in Russia for more than a decade, it is virtually unknown in this country. A great variety of problems to which it has been applied to obtain useful solutions includes an investigation of flexure and torsion of beams, a study of thermo-elastic stresses in composite cylinders, an analysis of deflection of anisotropic plates, and a multitude of problems characterized by the states of plane stress and plane strain.

Inasmuch as familiarity with the concepts of applied mathematics is a rare virtue, I shall reduce the use of the technical language to a minimum, and shall ask you to take for granted certain basic equations of the theory of elasticity. Failure to comprehend the origin of these equations will not impair the understanding of the general method of their solution.

We shall suppose that a two-dimensional region $R$, occupied by an elastic medium, is referred to a system of cartesian axes $(x, y)$. To fix the ideas we can think of the region $R$ as representing the cross-section of a long cylinder whose elements are parallel to the $z$-axis, and whose lateral surface is subjected to a distribution of external forces that is independent of the z-coordinate. Under the action of such forces, the medium, in general, will be distorted, and the displacement of the points of the region $R$ in the directions of the $x$ - and $y$ axes will be denoted by $u(x, y)$ and $v(x, y)$, respectively. If the medium

An address delivered before the meeting of the Society in Bethlehem, Pa., on December 30, 1941, by invitation of the Program Committee; received by the editors January $21,1942$. 
is assumed to be isotropic, then the stresses produced in the medium are connected with the derivatives of $u$ and $v$ by the formulas (Hooke's law)

$$
\tau_{x x}=\lambda \Delta+2 \mu \frac{\partial u}{\partial x}, \quad \tau_{y y}=\lambda \Delta+2 \mu \frac{\partial u}{\partial y}, \quad \tau_{x y}=\mu\left(\frac{\partial \vartheta}{\partial x}+\frac{\partial u}{\partial y}\right),
$$

where the $\tau$ 's are the components of the stress tensor, $\lambda$ and $\mu$ are elastic constants introduced by Lamé, and $\Delta \equiv \partial u / \partial x+\partial v / \partial y$.

If the medium is in equilibrium (in the absence of body forces) then the components of the stress tensor $\tau$ satisfy throughout the interior of the region the differential equations

$$
\frac{\partial \tau_{x x}}{\partial x}+\frac{\partial \tau_{x y}}{\partial y}=0, \quad \frac{\partial \tau_{x y}}{\partial x}+\frac{\partial \tau_{y y}}{\partial y}=0,
$$

while on the boundary $C$ of the region $R$ they fulfill the conditions

$$
\begin{aligned}
& \tau_{x x} \cos (x, v)+\tau_{x y} \cos (y, v)=X(s) \\
& \tau_{x y} \cos (x, v)+\tau_{y y} \cos (y, v)=Y(s),
\end{aligned}
$$

where $v$ denotes the exterior normal to the contour $C$, and $X$ and $Y$ are the $x$ - and $y$-components of the prescribed external force estimated per unit length $s$ of the contour $C$.

I shall call the problem of determining the solution of the system of equations (1.1), (1.2), (1.3), for the five unknown functions $\tau_{x x}$, $\tau_{x y}, \tau_{y y}, u, v$, the first boundary value problem of the plane theory of elasticity. Instead of specifying the distribution of external force on the boundary $C$ of the region $R$, we can impose the requirement that the displacements $u$ and $v$ assume prescribed values on the contour $C$. This latter problem will be referred to as the second boundary value problem.

The great majority of the two-dimensional boundary value problems in the theory of elasticity is reducible to the solution of these boundary value problems. We shall be concerned only with those solutions in which the components of the stress tensor are continuous and single-valued throughout the region $R$ together with their first and second partial derivatives with respect to $x$ and $y$. The displacements $u$ an $v$ will be assumed to be continuous and singlevalued in the region $R$ together with their partial derivatives including those of the third order. These restrictions arise essentially from physical considerations, and it is a consequence of the restrictions imposed on the displacements $u$ and $v$ that the components of the 
stress tensor determined from equations (1.2) satisfy in the region $R$ the Beltrami-Michell equation of compatibility, namely

$$
\nabla^{2}\left(\tau_{x x}+\tau_{y y}\right)=0
$$

where $\nabla^{2} \equiv \partial^{2} / \partial x^{2}+\partial^{2} / \partial y^{2}$.

It is clear that equations (1.2) represent a necessary and sufficient condition for the existence of a function $U(x, y)$, such that

$$
\tau_{x x}=\frac{\partial^{2} U}{\partial y^{2}}, \quad \tau_{y y}=\frac{\partial^{2} U}{\partial x^{2}}, \quad \tau_{x y}=-\frac{\partial^{2} U}{\partial x \partial y},
$$

and it follows at once from (1.4) that $U(x, y)$ satisfies the biharmonic equation

$$
\nabla^{2} \nabla^{2} U=0
$$

throughout the region $R$. The function $U$ is commonly known as Airy's stress function.

Substituting the relations (1.5) in the boundary conditions (1.3) and combining results lead to a compact boundary condition in the form

$$
\frac{d}{d s}\left(\frac{\partial U}{\partial y}-i \frac{\partial U}{\partial x}\right)=X+i Y,
$$

on $C$,

where $d / d s$ represents differentiation along the arc length of the contour $C$ and $i^{2}=-1$. Inasmuch as the right-hand member of (1.7) is a known function of the points of the contour $C$, it is clear that the solution of the first boundary value problem is reduced to the determination of the biharmonic function $U(x, y)$ whose derivatives, $\partial U / \partial x$ and $\partial U / \partial y$, are specified functions of the points of $C$. This latter problem was subject to a prize offered by the Paris Academy, and its complete theoretical solution for the case of a finite simplyconnected region $R$, bounded by a contour $C$ satisfying certain general conditions, was obtained by J. Hadamard and G. Lauricella. ${ }^{1}$ They solved the problem by reducing it to the solution of a Fredholm's integral equation.

A significant step in the direction. of the development of the theory was made by G. V. Kolossoff, whose Dorpat dissertation ${ }^{2}$ (1909) was concerned with the application of the theory of functions of a complex variable to the solution of plane problems of the theory of elasticity. Further important results were obtained by N. I. Mus-

1 Among the recipients of the prize were A. Korn, J. Hadamard, and G. Lauricella. See references [1], [2], and [3] at the end of this paper.

2 See [4-8]. 
chelišvili, who made use of conformal mapping and of integrals analogous to those of Cauchy to obtain an elegant solution of the first and second boundary value problems for an arbitrary simplyconnected domain (finite or infinite).

In a series of papers dating from 1919, Muschelišvili gave a detailed analysis of the character of solution in simply- and multiplyconnected domains and illustrated his method of solution by providing practically useful solutions of a number of specific problems [9-21].

The questions pertaining to the existence of solution in multiplyconnected domains were dealt with extensively by S. G. Michlin, who gave, in the period from 1933 to 1935 , several methods of theoretical solution of the first and second boundary value problems [22-26].

In the following section I shall limit myself to a discussion of Muschelišvili's method of solution of the boundary value problems of plane elasticity for simply-connected domains. While the application of this method to multiply-connected domains is important from a technical point of view (see $\$ 3$ ) the essence of the method is adequately illustrated by a consideration of the simpler case.

2. Reduction of the problem to a functional equation. It is clear from equation (1.6) that the function $\nabla^{2} U=P(x, y)$ is harmonic in $R$. Let $Q(x, y)$ denote the conjugate harmonic function, and introduce an analytic function $\phi(z)$ of a complex variable $z=x+i y$ defined by the formula

$$
P+i Q=4 \phi^{\prime}(z),
$$

where prime denotes the derivative with respect to $z$. If the real and imaginary parts of the function $\phi(z)$ are denoted by $p$ and $q$, respectively, then it is easily verified that

$$
\nabla^{2}(U-p x-q y)=0 .
$$

Accordingly, the function

$$
p_{1}=U-p x-q y
$$

can be considered as the real part of some analytic function $\chi(z)$, and since

$$
2(p x+q y) \equiv \bar{z} \phi(z)+z \overline{\phi(z)},
$$

one can write the biharmonic function $U$ in the form ${ }^{3}$

${ }^{3}$ The possibility of representing a biharmonic function with the aid of two analytic functions of a complex variable was noted first by $\mathbf{E}$. Goursat, Bulletin de la 


$$
2 U=\bar{z} \phi(z)+z \overline{\phi(z)}+\chi(z)+\overline{\chi(z)},
$$

where bars denote the conjugate complex values.

Inserting the value of $U$ given by (2.1) in the left-hand member of equation (1.7) yields the boundary condition in the form

$$
(X+i Y) d s=-i d\left[\phi(z)+z \overline{\phi^{\prime}(z)}+\overline{\psi(z)}\right],
$$

where $\psi(z)=\chi^{\prime}(z)$.

If both members of equation (2.2) are integrated along the contour $C$ from some arbitrary point $s=s_{0}$, there results

$$
\int_{s_{0}}^{s}(X+i Y) d s=-i\left[\phi(z)+z \overline{\phi^{\prime}(z)}+\overline{\psi(z)}\right]+\text { const., }
$$

so that the boundary condition can be written as

$$
\phi(z)+z \overline{\phi^{\prime}(z)}+\overline{\psi(z)}=f_{1}(s)+i f_{2}(s),
$$

on $C$,

where

$$
i \int_{s_{0}}^{s}(X+i Y) d s=f_{1}(s)+i f_{2}(s)
$$

The constant of integration has been omitted in the formula (2.3) since it can be fixed in an arbitrary manner without affecting the state of stress. ${ }^{4}$

A reference to the formulas (1.5) and (2.1) shows that the components of the stress tensor $\tau$ are easily calculable once the functions $\phi(z)$ and $\psi(z)$ are known, and it is readily checked by integrating equations (1.1) that

$$
2 \mu(u+i v)=-\left(\frac{\partial U}{\partial x}+i \frac{\partial U}{\partial y}\right)+\frac{2(\lambda+2 \mu)}{\lambda+\mu} \phi(z) .
$$

Thus the complete solution of the first boundary value problem is made to depend on the solution of the functional equation (2.3).

The formulation of the second boundary value problem in the form of a functional equation likewise presents no difficulty. In fact, the

Société Mathématique de France, vol. 26 (1898), p. 236. The derivation of Goursat's formula given above is due to N. I. Muschelišvili, Bulletin de l'Académie des Sciences de l' URSS, 1919, pp. 663-686. The analytic character of solutions of a biharmonic equation follows from this derivation, while the derivation of Goursat depends on the assumption that every biharmonic function is analytic.

${ }^{4}$ This statement follows from the fact that the state of stress, in a simply-connected domain, is not altered when the function $\phi(z)$ is replaced by $\phi(z)+c i z+\alpha+i \beta$ and $\psi(z)$ by $\psi(z)+\alpha^{\prime}+i \beta^{\prime}$, where $c, \alpha, \beta, \alpha^{\prime}$, and $\beta^{\prime}$ are arbitrary real constants. See, for example, reference [15] listed at the end of this paper. 
desired functional equation, in this case, follows almost at once from equation (2.4).

The limitations of time do not permit me to enter upon the discussion of the interesting questions connected with the existence and uniqueness of solution of the problem, and I shall confine myself to a remark that the proofs of uniqueness and existence of solution present no serious difficulties if one assumes the functions $\phi(z), \phi^{\prime}(z)$, and $\psi(z)$ to be continuous in the closed region $R$, the prescribed functions $X(s)$ and $Y(s)$ to satisfy Hölder's condition, and the contour $C$ to have a continuously turning tanget. ${ }^{5}$ It may be remarked that these restrictions are dictated by the behavior of stresses and displacement occurring normally in physical problems, and that they can be considerably relaxed by an analytically inclined mathematician.

I shall indicate in the remainder of this section some modes of attack on the problems that lead to the determination of the functions $\phi(z)$ and $\psi(z)$ in practically useful forms.

The solution of the functional equation (2.3) is almost immediate when the region $R$ is that bounded by a unit circle $|z| \leqq 1$. Since the functions $\phi(z)$ and $\psi(z)$ are analytic in $R$, one can write

$$
\phi(z)=\sum_{n=0}^{\infty} a_{n} z^{n}, \quad \psi(z)=\sum_{n=0}^{\infty} b_{n} z^{n}, \quad|z|<1 .
$$

The functions $X$ and $Y$, characterizing the prescribed distribution of stress on the boundary of the unit circle, can be regarded as functions of the polar angle $\theta$ defined by the relation $z=e^{i \theta}$. Then the right-hand member of (2.3) can be expressed in the form

$$
f_{1}+i f_{2}=i \int_{s_{0}}^{s}(X+i Y) d s=\sum_{-\infty}^{\infty} c_{n} e^{i n \theta},
$$

if one assumes that the functions $f_{1}$ and $f_{2}$ are of bounded variation. The Fourier coefficients $c_{n}$ being known, one can insert the foregoing expansion in the right-hand member of (2.3) and the series (2.5) in the left-hand member. A comparison of like powers of $e^{i \theta}$ on both sides of the resulting equation will yield expressions for the unknown coefficients $a_{n}$ and $b_{n}$ in terms of the known values $c_{n}$. The justification of the validity of the formal solution obtained in this manner presents no difficulty in most problems of practical interest, since the behavior of functions describing the specified distribution of stress on the boundary cannot be too pathological.

\footnotetext{
${ }^{5}$ See, for example, [26].
} 
If the contour $C$ bounding the region $R$ is not a circle, one can introduce a function

$$
z=\omega(\zeta)
$$

that maps the region $R$ conformally on the unit circle $|\zeta|<1$. Let the transforms of the functions $\phi(z)$ and $\psi(z)$ be denoted, respectively, by $\phi_{1}$ and $\psi_{1}$, so that $\phi_{1}(\zeta)=\phi[\omega(\zeta)]$ and $\psi_{1}(\zeta)=\psi[\omega(\zeta)]$. Then a simple calculation shows that the functional equation (2.3) goes over into

$$
\phi_{1}(\sigma)+\frac{\omega(\sigma)}{\overline{\omega^{\prime}(\sigma)}} \overline{\phi_{1}^{\prime}(\sigma)}+\overline{\psi_{1}(\sigma)}=f_{1}+i f_{2},
$$

where $\sigma=e^{i \theta}$ represents a point on the boundary $\gamma$ of the circle $|\zeta|=1$, and $f_{1}+i f_{2}$ is the transform of the right-hand member of (2.3) and hence can be regarded as a known function of $\theta$.

The method of solution in series indicated above can be applied to determine the analytic functions

$$
\phi_{1}(\zeta)=\sum_{n=0}^{\infty} a_{n} \zeta^{n}, \quad \psi_{1}(\zeta)=\sum_{n=0}^{\infty} b_{n} \zeta^{n}, \quad|\zeta|<1,
$$

and it is not difficult to verify that the coefficients $a_{n}$ and $b_{n}$ can be determined explicitly in terms of the Fourier coefficients of the functions $f_{1}+i f_{2}$ and $\omega(\sigma) / \overline{\omega^{\prime}(\sigma)}$, whenever the mapping function $\omega(\zeta)$ is a polynomial. ${ }^{6}$

If it is recalled that the mapping function for a suitably restricted simply-connected region can be approximated by a polynomial $z=\omega_{n}(\zeta)$ of sufficiently high degree (in the sense that the polynomial $z=\omega_{n}(\zeta)$ maps the region $|\zeta|<1$ on some region $R_{n}$ which can be made to approximate the region $R$ as closely as desired) then the practical usefulness of the method of solution outlined above becomes immediately obvious.

In a number of important problems the determination of the functions $\phi_{1}(\zeta)$ and $\psi_{1}(\zeta)$ can be greatly simplified by transforming the functional equation (2.6) into an equivalent pair of integral equations. Thus, consider the equation (2.6) and the equation

$$
\overline{\phi_{1}(\sigma)}+\frac{\overline{\omega(\sigma)}}{\omega^{\prime}(\sigma)} \phi_{1}^{\prime}(\sigma)+\psi_{1}(\sigma)=f_{1}-i f_{2}
$$

which is obtained from (2.6) by forming the conjugate expressions. If

${ }^{6}$ For further particulars see [13, pp. 287-296]. A detailed exposition and some specific calculations pertaining to several problems of physical interest are contained in [30, pp. 266-311]. 
both members of these equations are multiplied by $(1 / 2 \pi i) d \sigma /(\sigma-\zeta)$ and the resulting expressions integrated over the contour $\gamma$ of the unit circle, one obtains

$$
\begin{aligned}
\frac{1}{2 \pi i} \int_{\gamma} \frac{\phi_{1}(\sigma) d \sigma}{\sigma-\zeta} & +\frac{1}{2 \pi i} \int_{\gamma} \frac{\omega(\sigma)}{\overline{\omega^{\prime}(\sigma)}} \frac{\overline{\phi_{1}^{\prime}(\bar{\sigma})}}{\sigma-\zeta} d \sigma \\
& +\frac{1}{2 \pi i} \int_{\gamma} \frac{\overline{\psi_{1}(\sigma)}}{\sigma-\zeta} d \sigma=A(\zeta), \\
\frac{1}{2 \pi i} \int_{\gamma} \overline{\frac{\phi_{1}(\sigma)}{\sigma-\zeta}} d \sigma & +\frac{1}{2 \pi i} \int_{\gamma} \frac{\overline{\omega(\sigma)}}{\omega^{\prime}(\sigma)} \frac{\phi_{1}^{\prime}(\sigma)}{\sigma-\zeta} d \sigma \\
& +\frac{1}{2 \pi i} \int_{\gamma} \frac{\psi_{1}(\sigma)}{\sigma-\zeta} d \sigma=B(\zeta),
\end{aligned}
$$

where

$A(\zeta)=\frac{1}{2 \pi i} \int_{\gamma}\left[\left(f_{1}+i f_{2}\right) /(\sigma-\zeta)\right] d \sigma, B(\zeta)=\frac{1}{2 \pi i} \int_{\gamma}\left[\left(f_{1}-i f_{2}\right) /(\sigma-\zeta)\right] d \sigma$

The complete equivalence of the simultaneous equations (2.8) with (2.6) follows at once from the theorem of Harnack. The equations (2.8) can be simplified by applying Cauchy's integral formula, and by observing that

$$
\frac{1}{2 \pi i} \int_{\gamma} \frac{\overline{f(\sigma)}}{\sigma-\zeta} d \sigma=\overline{f(0)}
$$

where $f(\zeta)$ is any function continuous in the closed region $|\zeta| \leqq 1$ and analytic in the interior of the unit circle $\gamma$. Making use of these simplifications leads to a pair of integral equations

$$
\begin{aligned}
& \phi_{1}(\zeta)+\frac{1}{2 \pi i} \int_{\gamma} \frac{\omega(\sigma)}{\overline{\omega^{\prime}(\sigma)}} \frac{\overline{\phi_{1}^{\prime}(\sigma)}}{\sigma-\zeta} d \sigma+\overline{\psi_{1}(0)}=A(\zeta), \\
& \psi_{1}(\zeta)+\frac{1}{2 \pi i} \int_{\gamma} \frac{\overline{\omega(\sigma)}}{\omega^{\prime}(\sigma)} \frac{\phi_{1}^{\prime}(\sigma)}{\sigma-\zeta} d \sigma+\overline{\phi_{1}(0)}=B(\zeta) .
\end{aligned}
$$

It was demonstrated by Muschelišvili that the solution of these equations for the unknown functions $\phi_{1}(\zeta)$ and $\psi_{1}(\zeta)$ can be obtained in an elementary way in terms of the integrals of Cauchy's type whenever the mapping function $\omega(\zeta)$ is rational. ${ }^{7}$

7 See, for example, $[15,16]$. 
The system of integral equations (2.9) is not of the standard type, but it is possible to reduce the first of equations (2.9) to an ordinary integral equation of Fredholm, of the second kind. ${ }^{8}$ Once the function $\phi_{1}(\zeta)$ is determined from this equation, the second of equations (2.9) permits one to calculate the function $\psi_{1}(\zeta)$ by quadratures. Some effective methods of numerical solution of such equations were given recently by E. I. Nyström, D. I. Šerman, A. Gorgidze, A. Rukhadze and others [27-29].

3. Thermal stresses. One of the technically important problems is that of determining the state of stress produced in an elastic body by heating. Consider a body in the shape of a right cylinder whose length is large compared with the linear dimensions of the cross-section, and let the $x y$-plane of the cartesian coordinate system lie in one of the cross-sections of the cylinder. If such a body is subjected to a change in temperature which is a function of the $x$ - and $y$-coordinates alone, then the change of temperature will cause the body to expand. In general, the expansion of an elastic body cannot proceed freely and the body becomes stressed. The problem of determining the distribution of thermal stresses assumes particular importance in a study of compound columns. A concrete beam reenforced by steel and subjected to varying temperatures is a specific example.

It is not difficult to see that the determination of stresses in this case essentially reduces to the solution of a system of equations which differ from the system (1.1), (1.2), (1.3) only in that Hooke's law (1.1) is replaced by a more general one that takes into account the effect of thermal expansion on stresses. This generalized law has the form

$$
\begin{aligned}
\tau_{x x} & =\lambda \Delta+2 \mu \frac{\partial u}{\partial x}-(3 \lambda+2 \mu) \alpha T(x, y), \\
\tau_{y y} & =\lambda \Delta+2 \mu \frac{\partial v}{\partial y}-(3 \lambda+2 \mu) \alpha T(x, y), \\
\tau_{x y} & =\mu\left(\frac{\partial v}{\partial x}+\frac{\partial u}{\partial y}\right)
\end{aligned}
$$

B This reduction is accomplished essentially by differentiating both members of the equation with respect to $\zeta$ and by allowing $\zeta$ to approach $\sigma_{0}$, where $\sigma_{0}$ is an arbitrary point of the contour $\gamma$. The resulting equation has the form $\phi^{\prime}\left(\sigma_{0}\right)+(1 / 2 \pi i)$ $\int_{\gamma} K\left(\sigma_{0}, \sigma\right) \overline{\phi^{\prime}(\sigma)} d \sigma=F\left(\sigma_{0}\right)$, where $F\left(\sigma_{0}\right)$ is a known function and the kernel $K\left(\sigma_{0}, \sigma\right)$ is a continuous function provided that suitable restrictions are imposed on the mapping function $\omega(\zeta)$. See, for example, $[13,16]$. 
where $\alpha$ is the coefficient of the linear thermal expansion and $T(x, y)$ is the known change in temperature of the body.

If we introduce a stress function $U(x, y)$ defined by the formulas (1.5) and perform calculations analogous to those that led to the biharmonic equation (1.6), there will result the equation ${ }^{9}$

$$
\nabla^{2} \nabla^{2} U+k \nabla^{2} T=0
$$

where $k=2 \mu(3 \lambda+2 \mu)(\lambda+2 \mu)^{-1} \alpha$.

Setting $U=\Phi-V$, where $V$ is a suitable solution of the Poisson equation

$$
\nabla^{2} V=k T
$$

shows that equation (3.1) is equivalent to a system of two equations

$$
\nabla^{2} \nabla^{2} \Phi=0, \nabla^{2} V=k T .
$$

A calculation in every respect parallel to the one that led to the boundary condition (1.7) in this case yields

$$
\frac{d}{d s}\left(\frac{\partial \Phi}{\partial y}-i \frac{\partial \Phi}{\partial x}\right)=X+i Y+\frac{d}{d s}\left(\frac{\partial V}{\partial y}-i \frac{\partial V}{\partial x}\right), \quad \text { on } C,
$$

and it follows that the thermo-elastic problem is identical with the first boundary value problem discussed in $\$ \S 1$ and 2 .

An assertion of the mathematical equivalence of the two problems does not settle many specific questions regarding the behavior of cylinders when subjected to heat. The implications of the connection of the derivatives of the function $\Phi$ with those of the function $V$ as expressed in formula (3.2) were analyzed by B. E. Gatewood in a doctoral dissertation. ${ }^{10}$ Gatewood also studied the thermo-elastic problem for cylinders with longitudinal cavities and obtained some interesting results for cylinders composed of different materials. These results, however, are too specialized to be entered upon in this address.

It is obvious that a similar mode of attack can be expected to succeed in a study of thermal stresses in elastic plates made up of several layers of different materials. ${ }^{11}$

\footnotetext{
${ }^{9}$ Of course, the equation of compatibility, in this case, assumes a form different from (1.4).

10 Thermal stresses in long cylindrical bodies, dissertation, Wisconsin, 1939, $96 \mathrm{pp}$. See, also, B. E. Gatewood, this Bulletin, abstract 45-9-314. A paper based on this dissertation appears in Philosophical Magazine, (7), vol. 32 (1941), pp. 282-301.

${ }^{11}$ Some references to recent work on thermal stresses are given at the end of this paper. See [31-42].
} 
4. Deflection of non-isotropic plates. The industrial demands for lighter and stronger materials have given a new impetus to a study of the behavior of various structural members made of non-isotropic materials. In particular, the need of an accurate knowledge of the performance of plates and shells made of plywood is strongly felt, and in this connection some extensions of the method outlined in $\$ 2$ offer new possibilities. I shall indicate only one such extension which yields fruitful results in a study of small deflections of a thin non-isotropic plate subjected to a load $q(x, y)$ distributed normally over one of the faces of the plate. A very general type of anisotropy is assumed, and it will be supposed that the $x y$-plane of the coordinate system is the only plane of elastic symmetry.

It can be shown, ${ }^{12}$ by utilizing the usual assumptions of the thin plate theory, that the differential equation governing small deflection $w$ has the form:

$$
\begin{aligned}
b_{11} \frac{\partial^{4} w}{\partial x^{4}} & +3 b_{16} \frac{\partial^{4} w}{\partial x^{3} \partial y}+2\left(b_{12}+b_{66}\right) \frac{\partial^{4} w}{\partial x^{2} \partial y^{2}} \\
& +3 b_{26} \frac{\partial^{4} w}{\partial x \partial y^{3}}+b_{22} \frac{\partial^{4} w}{\partial y^{4}}=\frac{3 q(x, y)}{2 h^{3}},
\end{aligned}
$$

where the $b_{i j}$ are the elastic constants and $2 h$ is the thickness of the plate.

The equation (4.1) can be written in a symbolic form, with the aid of four linear operators, as

$$
D_{4} D_{3} D_{2} D_{1} w=\frac{3 q}{2 h^{3}},
$$

where $D_{i}=\partial / \partial y-\mu_{i} \partial / \partial x$, and the constants $\mu_{i}$ are the roots of the characteristic equation

$$
b_{22} \mu^{4}+3 b_{26} \mu^{3}+2\left(b_{12}+b_{66}\right) \mu^{2}+3 b_{16} \mu+b_{11}=0 .
$$

It follows from the fact that the potential energy of any physically realizable state of stress is positive that the roots $\mu_{i}$ of the characteristic equation (4.2) are complex, and since the coefficients $b_{i j}$ are real, they must have the forms $:^{13}$

$$
\mu_{1}=\alpha+\beta i, \quad \mu_{2}=\gamma+\delta i, \quad \mu_{3}=\bar{\mu}_{1}, \quad \mu_{4}=\bar{\mu}_{2},
$$

where $\alpha, \beta, \gamma, \delta$ are real and $\beta \neq 0, \delta \neq 0$.

${ }^{12}$ See $[43,44]$.

${ }_{13}$ A proof of this was given by S. N. Lechnitzky [43]. 
Accordingly, the general solution of equation (4.1), for the case of distinct roots, ${ }^{14}$ can be written as

$$
w=\sum_{k=1}^{4} F_{k}\left(x+\mu_{k} y\right)+w_{0}(x, y),
$$

where $w_{0}(x, y)$ is any particular solution of $(4.1)$, and the $F_{k}$ are arbitrary functions. Since the deflection $w(x, y)$ is real, it follows that the functions $F_{k}\left(x+\mu_{k} y\right)$ must be conjugate in pairs, so that one can write the solution (4.3) in the form:

$$
w=\phi\left(z_{1}\right)+\psi\left(z_{2}\right)+\overline{\phi\left(z_{1}\right)}+\overline{\psi\left(z_{2}\right)}+w_{0}(x, y),
$$

where $\phi\left(z_{1}\right)$ and $\psi\left(z_{2}\right)$ are arbitrary functions of the complex variables

$$
z_{1}=x+\mu_{1} y, \quad z_{2}=x+\mu_{2} y .
$$

Let the region corresponding to the middle surface of the unstrained plate be denoted by $R$ and introduce two new systems of cartesian coordinates $\left(x_{1}, y_{1}\right)$ and $\left(x_{2}, y_{2}\right)$ related to the coordinates $(x, y)$ by the formulas

$$
z_{1}=x_{1}+i y_{1}, \quad z_{2}=x_{2}+i y_{2} .
$$

It follows from inspection of (4.5) and (4.6) that the three systems of coordinates $(x, y),\left(x_{1}, y_{1}\right)$, and $\left(x_{2}, y_{2}\right)$ are connected with one another by the equations

$$
\begin{array}{ll}
x_{1}=x+\alpha y, & y_{1}=\beta y, \\
x_{2}=x+\gamma y, & y_{2}=\delta y .
\end{array}
$$

Hence to any point $P$ in the region $R$ there correspond points $P_{1}$ and $P_{2}$ in some regions $R_{1}$ and $R_{2}$ of the $z_{1}$ - and $z_{2}$-planes, respectively, and it is obvious that the regions $R_{1}$ and $R_{2}$ are obtainable from the region $R$ by a homogeneous deformation of the $x y$-plane. Thus, when the complex variable $z=x+i y$ varies in the region $R$, the variables $z_{1}$ and $z_{2}$ vary in the corresponding regions $R_{1}$ and $R_{2}$. Consequently, the functions $\phi\left(z_{1}\right)$ and $\psi\left(z_{2}\right)$ entering in the general solution (4.4) can be regarded as functions of the ordinary complex variables $z_{1}$ and $z_{2}$ defined in two different planes.

Ordinarily one is interested in determining the deflection $w$ when

14 In case of equal roots $\left(\mu_{1}=\mu_{2}, \mu_{3}=\mu_{4}\right)$ the general solution assumes the form $w=\left(x+\mu_{3} y\right) \quad F_{1}\left(x+\mu_{1} y\right)+\left(x+\mu_{1} y\right) \quad F_{2}\left(x+\mu_{3} y\right)+F_{3}\left(x+\mu_{1} y\right)+F_{4}\left(x+\mu_{3} y\right)+w_{0}(x, y)$ and a similar discussion can be carried out for this special case. If the plate is isotropic the equation (4.1) assumes the form $\nabla^{2} \nabla^{2} w=q / D$, where $D$ is the flexural rigidity, and the general solution can be written as $w=\phi(z)+\bar{z} \psi(z)+\overline{\phi(z)}+z \overline{\psi(z)}+w_{0}(x, y)$. 
the edges of the plate are subjected to a prescribed distribution of moments and shearing forces. Inasmuch as the moments and shearing forces are easily expressible in terms of the derivatives of the deflection function $w(x, y)$, one is led to a set of functional equations (appropriate to various modes of fixing plates at the edges) for the determination of the functions $\phi\left(z_{1}\right)$ and $\psi\left(z_{2}\right)$. In general, the functional equations are of the type

$$
a_{1 j} \phi^{\prime}\left(z_{1}\right)+a_{2 j} \psi^{\prime}\left(z_{2}\right)+\overline{a_{1 j} \phi^{\prime}\left(z_{1}\right)}+\overline{a_{2 j} \psi^{\prime}\left(z_{2}\right)}=A_{j}(s), \quad j=1,2,
$$

where the $a_{i j}$ are known constants and $A_{j}(s)$ are known functions prescribed on the contour $C$ bounding the region $R$.

The problem of determining the functions $\phi\left(z_{1}\right)$ and $\psi\left(z_{2}\right)$ in this case is naturally more involved than in the corresponding isotropic problem, but the complications are not of a basic sort. One practical mode of solving a system of equations (4.7) is to introduce a pair of suitable mapping functions $z_{1}=\omega_{1}(\zeta)$ and $z_{2}=\omega_{2}(\zeta)$ transforming the regions $R_{1}$ and $R_{2}$ into the unit circle $|\zeta| \leqq 1$ in the auxiliary $\zeta$-plane, and thereafter proceed in the same manner as indicated in $\$ 2$.

Some interesting results pertaining to the problem of deflection of a clamped non-isotropic plate, subjected to a quite general distribution of normal load, were obtained recently by V. Morkovin in a partially completed doctoral dissertation. By utilizing the method outlined above, Morkovin obtained in closed form the solution of the problem of deflection of a clamped elliptical plate when the loading function $q(x, y)$ is a polynomial in $x$ and $y$. The important case of a clamped circular plate follows directly from it by specializing the values of the parameters appearing in the solution.

5. Concluding remarks. The foregoing account of a somewhat novel use of the theory of functions of a complex variable was concerned mainly with the applications to two-dimensional problems of elasticity. However, the central idea of replacing the differential equation and boundary conditions by an equivalent functional equation is obviously capable of extension to problems in other branches of applied mathematics.

A consideration of the problems of equilibrium of cylindrical bodies, and especially those relating to a study of initially curved and twisted beams, shows that occasionally there are advantages in utilizing similar notions in some classes of three-dimensional problems [4856].

The importance of the theory of functions of a complex variable in connection with the solution of the boundary value problems in- 
volving Laplace's equation is, of course, recognized by every applied mathematician. However, even in the consideration of such problems as those of Neumann and Dirichlet, the engineers appear to be so absorbed in special devices and artifices that a simple formulation of such problems is often overlooked.

In order to set in relief the basic notions that underlie the foregoing formulation of the two-dimensional problems of elasticity, it may not be out of place to recall a simple approach to the problem of Dirichlet.

Let the function $U(x, y)$, continuous in the closed simply-connected region $R$ and harmonic in the interior of $R$, assume continuous values $f(s)$ on the boundary $C$ of $R$. Then the boundary condition can be written in the form

$$
\phi(z)+\overline{\phi(z)}=2 f(s) \quad \text { on } C,
$$

where $\operatorname{Re} \phi(z)=U(x, y)$. Now, if the region $R$ is mapped conformally on the unit circle $|\zeta| \leqq 1$ with the aid of the function $z=\omega(\zeta)$, then the foregoing boundary condition assumes the form

$$
\phi_{1}(\sigma)+\overline{\phi_{1}(\sigma)}=2 f_{1}(\theta),
$$

where $\sigma=e^{i \theta}$, and $\phi_{1}(\zeta)$ and $f_{1}(\theta)$ are the transforms of $\phi(z)$ and $f(s)$. Multiplying by $(1 / 2 \pi i) d \sigma /(\sigma-\zeta)$ and integrating over the contour $\gamma$, yields

$$
\frac{1}{2 \pi i} \int_{\gamma} \frac{\phi_{1}(\sigma)}{\sigma-\zeta} d \sigma+\frac{1}{2 \pi i} \int_{\gamma} \frac{\overline{\phi_{1}(\sigma)}}{\sigma-\zeta} d \sigma=\frac{1}{\pi i} \int_{\gamma} \frac{f_{1}(\theta)}{\sigma-\zeta} d \sigma,
$$

and the evaluation of integrals gives at once

$$
\phi_{1}(\zeta)=\frac{1}{\pi i} \int_{\gamma} \frac{f_{1}(\theta)}{\sigma-\zeta} d \sigma+\text { const. }
$$

which is merely the formula of Schwarz.

The remarkable simplicity of the formulation of the two-dimensional problems of elasticity with the aid of functional equations is likely to produce an impression that the task of obtaining practically useful solutions is a straightforward matter. While it is true that in some cases the solution of rather difficult problems is obtained with remarkable ease and rapidity, in many instances severe calculational difficulties are present. These difficulties are connected mainly with the determination of the mapping function. However, recent advances in the development of practical methods of constructing map- 
ping functions for simply- and multiply-connected domains justify some degree of optimism.

\section{REFERENCES}

1. A. Korn, Sur les équations de l'élasticité, Annales de l'École Normale Supérieure, vol. 24 (1907), pp. 9-75.

2. J. Hadamard, Mémoire sur le problème d'analyse relatif à l'équilibre des plaques élastiques encastrêes, Mémoires des Savants Etranger, vol. 33 (1908), 128 pp.

3. G. Lauricella, Sur l'intégration de l'équation relative à l'équilibre des plaques elastiques encastrées, Acta Mathematica, vol. 32 (1909), pp. 202-256.

4. G. V. Kolossoff, Sur les problèmes d'élasticité d' deux dimensions, Comptes Rendus de l'Académie des Sciences, Paris, vol. 146 (1908), pp. 522-525.

5. ——, ibid., vol. 148 (1909), pp. 1242-1244, 1706.

6. - On an application of the theory of functions of a complex variable to plane problems of the mathematical theory of elasticity, dissertation in Russian, Dorpat, 1909.

7. —— Über einige Eigenschaften des ebenen Problems der Elastizitätstheorie, Zeitschrift für Mathematik und Physik, vol. 62 (1914), pp. 383-409.

8. - Application of a Complex Variable to Theory of Elasticity (in Russian), Moscow-Leningrad, 1935, 224 pp.

9. N. I. Muschelišvili, Sur l'intégration de l'équation biharmonique, Bulletin de l'Académie des Sciences de l'URSS, 1919, pp. 663-686.

10. —- Applications des intégrales analogues à celles de Cauchy à quelques problèmes de la Physique Mathématique, Tillis Université, 1922, 157 pp.

11. - Sur l'intégration approchée de l'équation biharmonique, Comptes Rendus de l'Académie des Sciences, Paris, vol. 185 (1927), pp. 1184-1186.

12. - Sur la solution du problème biharmonique pour l'aire exterieure a une ellipse, Mathematische Zeitschrift, vol. 26 (1927), pp. 700-705.

13. - Nouvelle méthode de réduction du problème biharmonique fondamental à une équation de Fredholm, Comptes Rendus de l'Académie des Sciences, Paris, vol. 192 (1931), pp. 77-79.

14. —- Théorèmes d'existance relatifs au problème biharmonique et aux problèmes d'élasticité à deux dimensions, ibid., pp. 221-223.

15. —- Recherches sur les problèmes aux limites relatifs à l'équation biharmonique et aux équations de l'elasticité à deux dimensions, Mathematische Annalen, vol. 107 (1932), pp. 282-312.

16. - - Praktische Lösung der fundamentalen Randwertaufgaben der Elastizitätstheorie in der Ebene für einige Berandungsformen, Zeitschrift für angewandte Mathematik und Mechanik, vol. 13 (1933), pp. 264-282.

17. - - Solution of a plane problem of elasticity for an ellipse (in Russian), Journal of Applied Mathematics and Mechanics, vol. 1 (1933), pp. 5-12.

18. - Nouvelle méthode générale pour résoudre les problèmes fondamentaux aux limites de la thêorie de l'élasticité à deux dimensions, Comptes Rendus de l'Académie des Sciences de l'URSS, vol. 3 (1934), pp. 7-11.

19. - Discussion des nouvelles équations intégrales de la thêorie de l'élasticitê à deux dimensions, ibid., pp. 73-77.

20. ——, Quelques problèmes fondamentaux de la théorie mathématique de l'élasticité (in Russian), Publication de l'Académie des Sciences de l'URSS, 1935, 453 pp.

21. I. Malkin, Über einige neuere Arbeiten auf dem Gebiete der Elastizitätslehre, Zeitschrift für angewandte Mathematik und Mechanik, vol. 10 (1930), pp. 182-197. 
22. S. G. Michlin, Le problème biharmonique fondamental d deux dimensions, Comptes Rendus de l'Académie des Sciences, Paris, vol. 197 (1933), pp. 608-610.

23. - Reduction of a plane problem of the theory of elasticity to an integral equation (in Russian), Comptes Rendus de l'Académie des Sciences de l'URSS, vol. 1 (1934), pp. 295-301.

24. - Quelques remarques relatives à la solution des problèmes plans d'élasticité, Mathematičeski Sbornik, vol. 41 (1934), pp. 408-414.

25. - Theorem of uniqueness for a fundamental biharmonic problem (in Russian), ibid. pp. 284-291.

26. - Le problème plan de la théorie statique d'élasticitê, Publications de l'institut seismologique, no. 65, Académie des Sciences de l'URSS, 1935, 82 pp.

27. E. I. Nyström, Acta Mathematica, vol. 54 (1930), pp. 185-204.

28. D. I. Serman, Publications de l'institut seismologique, no. 86 , Académie des Sciences de l'URSS, 1938, 50 pp.

29. A. Gorgidze and A. Rukhadze, Mitteilungen der Georgischen Abteilung der Akademie der Wissenschaften der USSR, vol. 1 (1940), pp. 255-258.

30. I. S. Sokolnikoff, Mathematical Theory of Elasticity, Brown University 1941, 394 pp.

31. N. Lebedew, Über die Warmespannungen in Elastizitätstheorie (in Russian), Journal of Applied Mathematics and Mechanics, vol. 2 (1934), pp. 52-60.

32. H. Poritsky, Analysis of thermal stresses in sealed cylinders and the effect of viscous flow during anneal, Physics, vol. 5 (1934), pp. 406-411.

33. J. L. Maulbetsch, Thermal stresses in plates, Journal of Applied Mechanics, Transactions of the American Society of Mechanical Engineers, vol. 57 (1935), pp. A-141-146.

34. K. Marguerre, Thermoelastische Platten-Gleichungen, Zeitschrift für angewandte Mathematik und Mechanik, vol. 15 (1935), pp. 369-372.

35. M. A. Biot, A general property of two-dimensional thermal stress distribution, Philosophical Magazine, vol. 19 (1935), pp. 540-544.

36. J. N. Goodier, The thermal stress in a strip due to variation of temperature along the length and through the thickness, Physics, vol. 7 (1936), pp. 156-159.

37. N. Lebedew, Thermal stresses in circular ring (in Russian), Journal of Applied Mathematics and Mechanics, vol. 3 (1936), pp. 76-84.

38. J. N. Goodier, Thermal stresses, Journal of Applied Mechanics, Transactions of the American Society of Mechanical Engineers, vol. 59 (1937), pp. A-33-36.

39. - On the integration of the thermo-elastic equations, Philosophical Magazine, vol. 23 (1937), pp. 1017-1032.

40. H. Poritsky, Thermal stresses in cylindrical pipes, ibid., vol. 24 (1937), pp. 209223.

41. I. S. Sokolnikoff and E. S. Sokolnikoff, Thermal stresses in elastic plates, Transactions of this Society, vol. 45 (1939), pp. 235-255.

42. B. E. Gatewood, Thermal stresses in long cylindrical bodies, dissertation, Wisconsin, 1939, 96 pp.

43. S. G. Lechnitzky, Über einige Fragen der Biegungstheorie der dïnnen Platten (in Russian), Journal of Applied Mathematics and Mechanics, (n.s.), vol. 2 (1939), pp. 181-210.

44. - Biegung nichthomogener anisotropen symmetrisch anfgebauter dïnner Platten (in Russian), ibid., vol. 5 (1941), pp. 71-92.

45. A. I. Lourye, Problems concerning the bending of a circular plate (in Russian), ibid., vol. 4 (1940), pp. 93-102. 
46. M. M. Friedman, Über einige Fragen der Theorie der Biegung von dïnnen isotropen Platten (in Russian), Journal of Applied Mathematics and Mechanics, vol. 5 (1941), pp. 93-102.

47. I. S. Sokolnikoff, Deflection of non-isotropic elastic plates, this Bulletin, abstract 47-5-257.

48. N. I. Muschelišvili, Sur le problème de torsion des cylindres élastiques isotropes, Rendiconti, R. Academia dei Lincei, vol. 9 (1929), pp. 295-300.

49. - Sur le problème de torsion des poutres élastiques composées, Comptes Rendus de l'Académie des Sciences, Paris, vol. 194 (1932), p. 1435.

50. - On the problem of torsion and bending of compound beams (in Russian) Bulletin de l'Académie des Sciences de l'URSS, vol. 5 (1932), pp. 907-945.

51. I. S. Sokolnikoff and E. S. Sokolnikoff, Torsion of regions bounded by circular arcs, this Bulletin, vol. 44 (1938), pp. 384-387.

52. D. B. Panov, Torsion of nearly prismatic beams, Comptes Rendus de l'Académie des Sciences de l'URSS, vol. 20 (1938), pp. 251-253.

53. P. M. Riz, Deformations of naturally twisted bars, ibid., vol. 23 (1939), pp. 17$20 ; 441-444 ; 765-767$.

54. - Deformations of bars with slightly curved axis, ibid., vol. 24 (1939), pp. $110-113 ; 229-231$.

55. A. K. Ruchadze, Problem of bending of nearly prismatic beams (in Russian), Mitteilungen der Georgischen Abteilung der Akademie der Wissenschaften der USSR, vol. 1 (1940), pp. 577-581.

56. - On the problem of deformation of a beam with slightly curved axis (in Russian), ibid., vol. 2 (1941), pp. 35-42.

57. Hajimu Okubo, Stress systems in an aelotropic rectangular plate, Zeitschrift für angewandte Mathematik und Mechanik, vol. 21 (1941), pp. 162-176.

UNIVERSITY OF WISCONSIN 